Research Article

\title{
Study of Transformer Core Vibration and Noise Generation Mechanism Induced by Magnetostriction of Grain-Oriented Silicon Steel Sheet
}

\author{
He Qiang ${ }^{(1)},{ }^{1}$ Nie Jingkai, ${ }^{1}$ Zhang Songyang, ${ }^{2}$ Xiao Weimin, ${ }^{3}$ Ji Shengchang, ${ }^{4}$ and Chen Xin ${ }^{1}$ \\ ${ }^{1}$ State Key Laboratory of Advanced Power Transmission Technology, Global Energy Interconnection Research Institute Co.Ltd., \\ Beijing 102201, China \\ ${ }^{2}$ State Grid Henan Electric Power Research Institute, Zhengzhou 450052, China \\ ${ }^{3}$ Beijing Key Laboratory of Environment Noise and Vibration, Beijing Municipal Institute of Labor Protection, Beijing 100054, \\ China \\ ${ }^{4}$ State Key Laboratory of Electrical Insulation and Power Equipment, Xi'an Jiaotong University, Xi'an 710049, China
}

Correspondence should be addressed to He Qiang; heqiang0927@163.com

Received 15 July 2020; Revised 11 April 2021; Accepted 21 April 2021; Published 4 May 2021

Academic Editor: Ricardo A. Ramirez-Mendoza

Copyright (c) $2021 \mathrm{He}$ Qiang et al. This is an open access article distributed under the Creative Commons Attribution License, which permits unrestricted use, distribution, and reproduction in any medium, provided the original work is properly cited.

The problem of vibration and noise in the iron core of power transformers remains quite topical. Insofar as the state-of-the-art iron core and binding production adopt the lamination method and weft-free adhesive tape, respectively, the transformer core vibration is mainly attributed to the silicon steel sheet (SSS) magnetostriction. In this paper, based on the magnetostriction of grain-oriented SSS, an in-depth analysis of the vibration generation mechanism in the transformer core was performed. The SSS microstructure was observed, its magnetostrictive properties at different magnetic flux densities were tested, and a core-simulating four-corner iron core model was constructed to analyze the vibration characteristics. Modal, vibration, and noise tests were performed on an actual $110 \mathrm{kV}$ transformer core under no-load conditions. The results show that the core vibration is related to SSS's deformation mechanism. The vibration magnitude in different core parts varies due to the magnetostriction anisotropy. The vibration in vertical to the core plane is the largest, and its magnitude in the core center is lower than those at the seams in the same plane. The core vibration and noise exhibit a significant correlation, while modal characteristics strongly influence the core vibration and noise intensity.

\section{Introduction}

Power transformers are critical integral parts of the power grid system, and their safe operation is mandatory for the entire system's reliability. The vibration of transformers affects the power equipment's normal operation, service life, and reliability, while the vibration and noise also have a negative impact on human health and comfort [1-3]. The transformer vibration has two main constituents: (i) winding vibration caused by the electromagnetic force generated by the interaction of the current in a winding with leakage flux, and (ii) iron core vibration caused by the magnetostriction forces in the silicon steel sheet (SSS). Under no-load conditions, there is no current in the winding, and the transformer vibration is mainly generated by the core. The vibration signal measured at this time is the core vibration signal, which is induced by the SSS magnetostriction.

So far, most research on the core vibration of transformers is mainly focused on magnetostrictive properties of materials [4-6], core vibration, noise testing, vibration transmission characteristics [7-17], and theoretical or numerical modeling [18-26]. There are also some studies focused on signal acquisition for vibration analysis and feature recognition [27-32]. Cheng et al. [4] studied magnetic and magnetostrictive properties of different grades of SSS and proposed the selection of grain-oriented SSS in core materials. Wang et al. [6] studied the magnetostrictive dynamic hysteresis characteristics of a grain-oriented electrical steel 
under different elliptical magnetization loci based on a rotational magnetic properties tester. Moses et al. [7] carried out experimental tests on the surface vibration and noise of transformer cores using a laser vibrometer. Okabe et al. [8] studied the vibration characteristics and mode shapes of the three-phase transformer core under different excitations and analyzed the reasons for the difference between the frequency spectrum and noise spectrum of the magnetostrictive vibration under partial frequency excitation. Ma et al. [15] carried out vibration and noise tests on the inside and outside of a typical $10 \mathrm{kV}$ distribution transformer tank and acquired the acoustic-vibration characteristic and propagation law of the $10 \mathrm{kV}$ distribution transformer. Zhang et al. [16] used the magnetostrictive orthogonal calculation method to simulate the vibration of iron cores of the transformer and shunt reactor models. The calculation and test results showed that Maxwell stress was the main reason of reactor core vibration, and magnetostriction was the main reason of the transformer core. Liu et al. [18] proposed a transformer vibration transmission model based on motion transmission. Through experiments and theoretical calculations, the accuracy of the vibration transmission method was verified, which could be used to analyze the vibration relationship between the transformer core and the casing. Javorski et al. [20] established a calculation method of magnetostrictive force based on experiments and analyzed the magnetostriction of laminated cores in different directions. Ghalamestani et al. [22] calculated the core deformations of a three-phase transformer under a purely sinusoidal magnetization with $50 \mathrm{~Hz}$ frequency and a magnetisation with a fifth harmonic component by an twodimensional (2D) finite element technique. Hu et al. [23] calculated the sound pressure level of the radiated noise around the transformer based on the established finite element model combining transient electromagnetic field analysis, mechanical field analysis, and acoustic analysis. Comparison of calculated results and measured data verified that the combined noise-calculating model was applicable for transformer noise prediction. Zhang et al. [24] studied a fast and accurate calculation method for the core vibration of a DFP-270000/500 type super high-voltage generator transformer. Based on the vibration acoustic coupling calculation, the damping coefficient of the core vibration of the transformer was obtained, and the magnetic structural coupling analysis was used for the vibration of the transformer core. Liu et al. [25] established a complete fully coupling modeling chain of such a transformer, from threephase magnetic flux injection to the $2 \mathrm{D}$ deformation and acoustic power estimation of the transformer core, taking into account the phase current coupling, magnetic flux excitation (voltage excitation), material anisotropy, magnetostrictive-induced stress, and multilayer homogenization. However, most of the above works are single-stage studies, ignoring the transfer between each stage. Therefore, the mechanism of core vibration generation has not been comprehensively described yet.

In this paper, according to the evolution process from the material to the product, the microstructure and magnetostrictive properties of grain-oriented SSS are tested, and a four-corner model of the core is built to simulate the first-order vibration of the core. Next, the modal, vibration, and noise tests of the $110 \mathrm{kV}$ transformer core product are performed. The in-depth analysis of the mechanism of core vibration generation can provide design ideas and data support for the preparation of low-noise cores.

\section{Mechanism of Iron Core Vibration}

The core vibration is mainly caused by the magnetostrictive phenomenon of the SSS and the Maxwell electromagnetic force. Due to the continuous improvement of the lamination method used for the iron core manufacturing and the weftfree adhesive tape application in its binding, the transformer core vibration is mainly controlled by the SSS magnetostriction. When the SSS is subjected to an external magnetic field, its dimensions undergo contraction and/or elongation and recover to the initial values after the external magnetic field removal. This phenomenon is referred to as the magnetostrictive effect.

The magnetostriction ratio is commonly used to reflect magnetostriction, which can be expressed as

$$
\varepsilon=\frac{\Delta L}{L},
$$

where $\varepsilon$ is the SSS axial magnetostriction ratio, $\Delta L$ is the SSS maximum axial expansion or contraction, and $L$ is the SSS original axial dimension.

Assuming the supply voltage is $U_{1}=U_{s} \sin \omega t$, according to the principle of electromagnetic induction, the magnetic induction intensity in the core is

$$
B=\frac{\phi}{S}=\frac{U_{s}}{\omega N S} \cos \omega t=B_{0} \cos \omega t,
$$

where $\varphi$ is the core magnetic flux, $S$ is the core crosssectional area, and $B_{0}=\left(U_{s} / \omega N S\right)$ is the magnitude of the magnetic induction intensity.

When the iron core works in an unsaturated state, the magnetic flux density and the magnetic field strength have a linear relationship. The magnetic field strength in the iron core is

$$
H=\frac{B}{\mu}=B \frac{H_{c}}{B_{s}}=B_{0} H_{c} \frac{\cos \omega t}{B_{s}},
$$

where $B_{s}$ is the saturation magnetic induction of the core and $H_{c}$ is the coercive force.

Under the effect of an external magnetic field, a smallscale deformation of the SSS caused by magnetostriction satisfies the following relationship:

$$
\frac{\Delta L}{L} \frac{1}{\mathrm{~d} H}=|H| \frac{2 \varepsilon_{s}}{H_{c}^{2}},
$$

where $\varepsilon_{s}$ is the saturation magnetostriction ratio of the SSS.

Based on the above relationship, one can derive the maximum axial elongation of the core caused by the SSS magnetostriction as follows: 


$$
\Delta L=L \int_{0}^{H}|H| \frac{2 \varepsilon_{s}}{H_{c}^{2}} \mathrm{~d} H=L \frac{\varepsilon_{s} H^{2}}{H_{c}^{2}}=L \frac{\varepsilon_{s} B^{2}}{B_{s}^{2}}=\frac{L \varepsilon_{s}^{2} U_{s}^{2}}{\left(\omega N_{\text {core }} S B_{s}\right)^{2}} \cos ^{2} \omega t
$$

Therefore, when the transformer is unloaded, the core vibration acceleration caused by the SSS magnetostriction is

$$
a=\frac{\mathrm{d}^{2} \Delta L}{\mathrm{~d} t^{2}}=-\frac{2 L \varepsilon_{s} U_{s}^{2}}{\left(N_{\text {core }} S B_{s}\right)^{2}} \cos 2 \omega t .
$$

From equation (6), it can be deduced that the vibration acceleration is proportional to the square of the voltage if the core material and operating temperature remain unchanged:

$$
a_{c} \propto u_{s}^{2}
$$

The magnetostriction variation period is twice lower than that of the alternating electromagnetic field, while the fundamental frequency of the core vibration caused by the magnetostriction is twice higher than the electromagnetic field variation frequency $\omega$.

However, in practice, the waveform of the core magnetic flux density is not a standard sine wave. In addition, the magnetic flux distributions and paths of the core outer and inner frames are different, which causes the core vibration signal to contain a large number of higher harmonic components, besides the fundamental frequency component.

\section{Materials and Methods}

(1) Materials: the grain-oriented silicon steel sheet (SSS) material used in the test was the B30P105 cold-rolled finished product produced by Baoshan Iron \& Steel Co., Ltd., China.

(2) Grain-oriented SSS microstructure measurement: microstructure and texture analysis were the basic methods to evaluate grain-oriented SSS. The grainoriented SSS was eroded by hot hydrochloric acid solution and then photographed. The texture was measured with electron backscattered diffractometer (EBSD) attached to Zeiss Ultra 55 field emission scanning electron microscope.

(3) Grain-oriented SSS magnetostriction measurement: MST500 magnetostriction measurement system was used to test the magnetostriction coefficient and A-weighted velocity (AWV) noise of grain-oriented SSS according to IEC/TR 62581-2010. The sample dimensions are $100 \mathrm{~mm} \times 600 \mathrm{~mm}$. In the measuring process, one end of the sample is fixed by the clamping part of the device, and the other end of the sample is in the free state. The resolution of the laser is $10 \mathrm{~nm} / \mathrm{m}$. A photo of the magnetostriction coefficient measuring instrument is shown in Figure 1.

(4) Four-corner core test model: a four-corner iron core model was established to simulate the first-order vibration of the transformer core. The four-corner iron core model adopted a three-step stepwise overlap method; two pieces were stacked on one another and connected by a $45^{\circ}$ diagonal joint. The winding was evenly wound on the two arms and was used in series. There was a total of 220 turns, $800 \mathrm{~mm}$ in length, $100 \mathrm{~mm}$ in width, and $30 \mathrm{~mm}$ in stack thickness. The acoustic vibration analysis system by the B \& K Company, Denmark, was used to collect the vibration signals at the five measurement points in the central area and the seam area of the parallel and vertical core planes, as shown in Figure 2.

(5) Transformer core measurement: the actual product used a three-phase dual-winding 50MVA $110 \mathrm{kV}$ transformer core to test its vibration and noise characteristics and natural modes. The sound level meter and acoustic vibration analysis system by the $B$ \& K Company, Denmark, were used for sound vibration measurement and analysis under the no-load condition, and the modal test of the iron core was performed via a force impact hammer and accelerometer. The noise and vibration measurement points are shown in Figure 3.

\section{Results and Discussion}

4.1. Microstructure and Magnetostriction Properties of GrainOriented SSS. Figures 4 and 5 show the microstructure and texture of different regions of the SSS, respectively. It can be seen that microstructures of the central and edge seam areas of both regions of interest are highly grain-oriented silicon steel that have been processed by cold rolling and annealing. The texture structure characteristics are consistent. Complete secondary recrystallization occurred in both areas, resulting in $\mathrm{cm}$-sized grains with irregular morphology and different sizes. The crystal orientation distribution of both regions is uniform. According to $\{200\}$ pole figure observation results, both areas are sharp Goss textures. The Goss orientation deviation angle is small, and there are no grainoriented grains that deteriorate magnetic properties such as $\{110\}\langle 227\rangle$ and $\{110\}\langle 112\rangle$.

Figure 6 plots the magnetostriction butterfly curve of the SSS in the rolling direction under a $50 \mathrm{~Hz}$ sine period excitation magnetic field. It can be seen that the butterfly curve has left-right symmetry. Its "wing" opening faces downwards, and magnetostriction has both elongation and contraction strains, the latter prevailing over the former ones. This prevalence may be due to the dynamic motion of the domain wall or the re-organization of the magnetic domain structure. Magnetostrictive shrinkage is caused by a $90^{\circ}$ unfavorable magnetic domain's magnetic moment rotation. With an increase in the magnetic flux density, the $90^{\circ}$ magnetic domain volume is continuously reduced. When the maximum magnetic flux density reaches $1.7 \mathrm{~T}$, the magnetostriction will no longer increase monotonously and start to drop. The butterfly curve wings narrowed, and the magnetostriction tended to saturation.

The magnetostrictive single-value curves under different magnetic densities are shown in Figure 7. It can be seen that, with an increase in the applied magnetic field intensity, the magnetostrictive peak-to-peak value $\lambda_{p-p}$, which is defined as the difference between $\lambda_{p+}$ and $\lambda_{p^{-}}$values under 


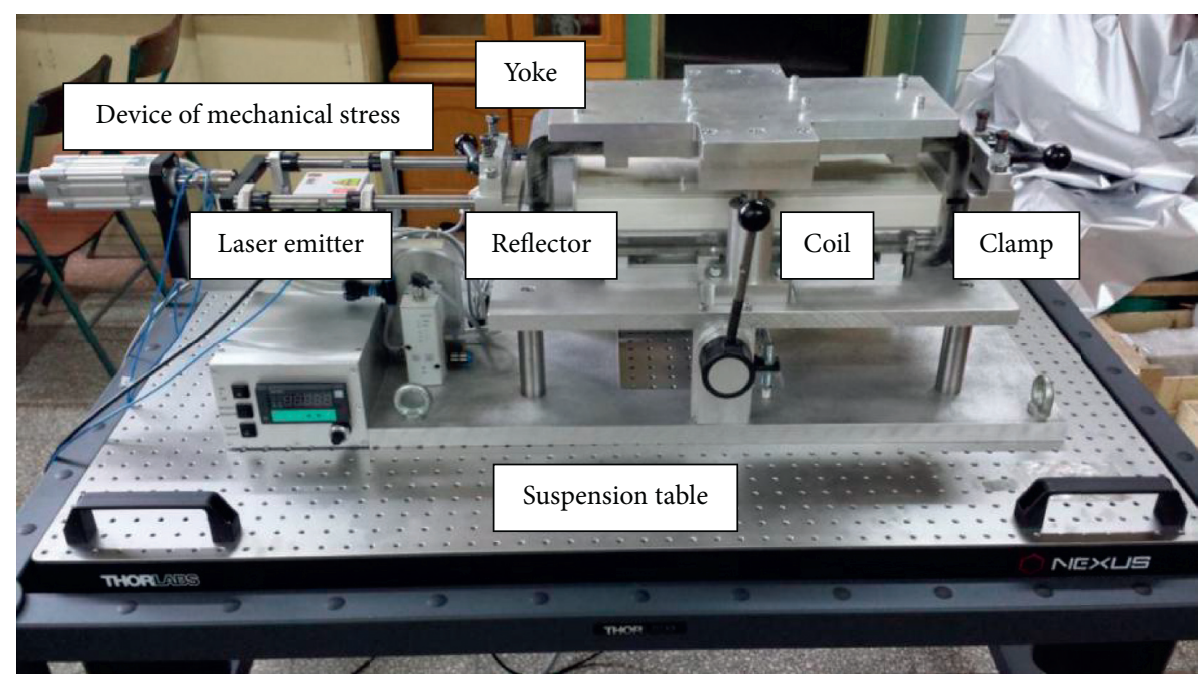

Figure 1: Photo of the magnetostriction coefficient measuring instrument.

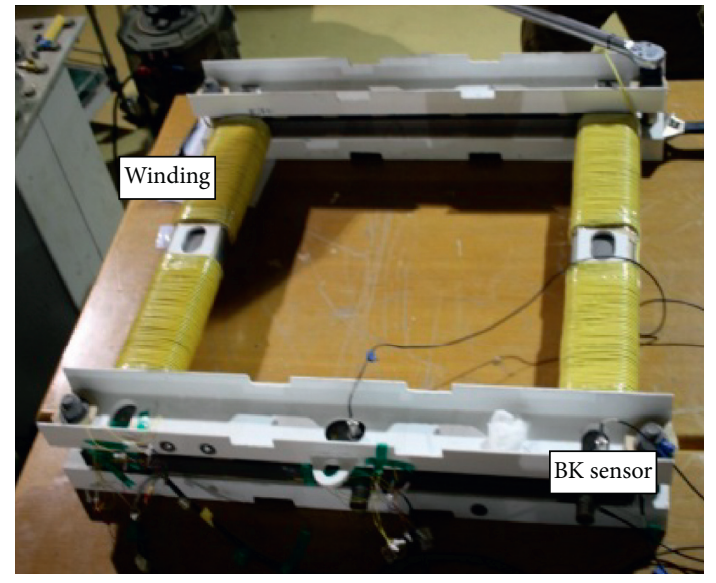

(a)

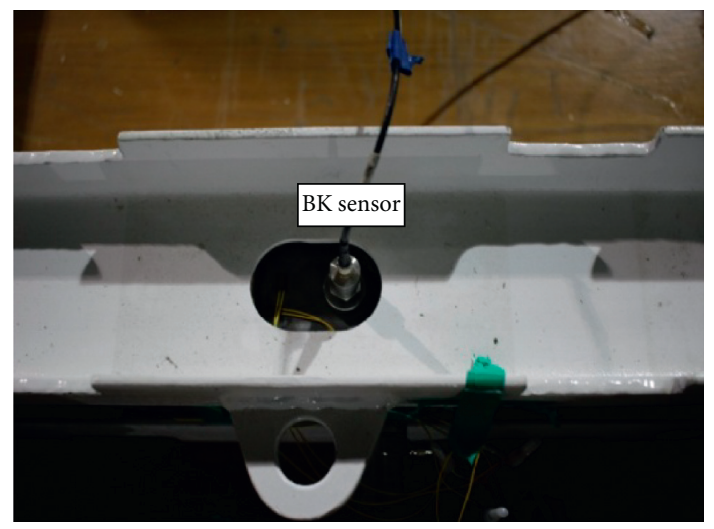

(c)

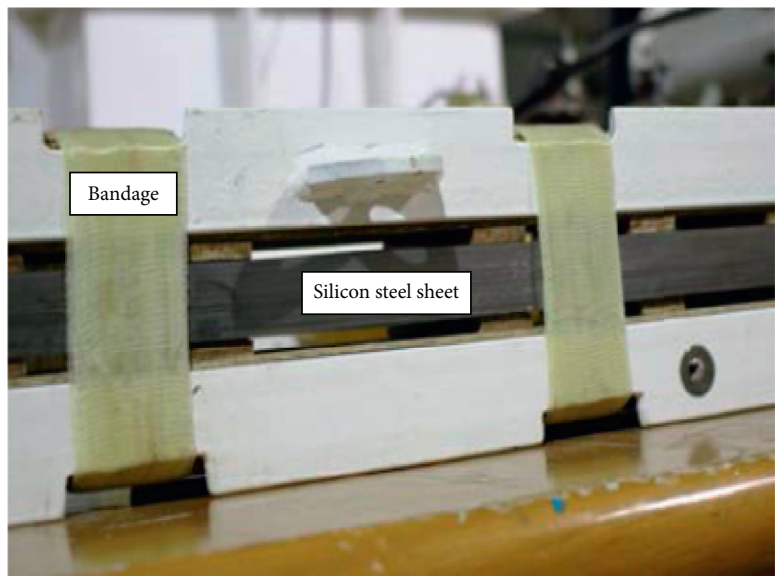

(b)

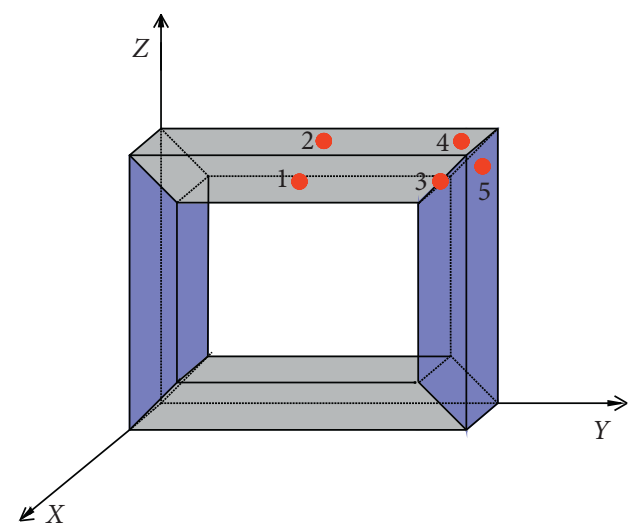

(d)

Figure 2: Four-corner iron core model test platform. (a) Top view. (b) Side view. (c) Vibration sensor. (d) Acceleration measurement point.

alternating magnetization, gradually increases. When the material magnetic properties tend to be saturated, the magnetostriction also appears to be saturated. From a microscopic perspective, the magnetic domains constituting the material are gradually arranged in parallel to the external field direction under this field action, and the magnetization process also changes with the magnetic domain size. When all magnetic domains form a single domain sample, the 


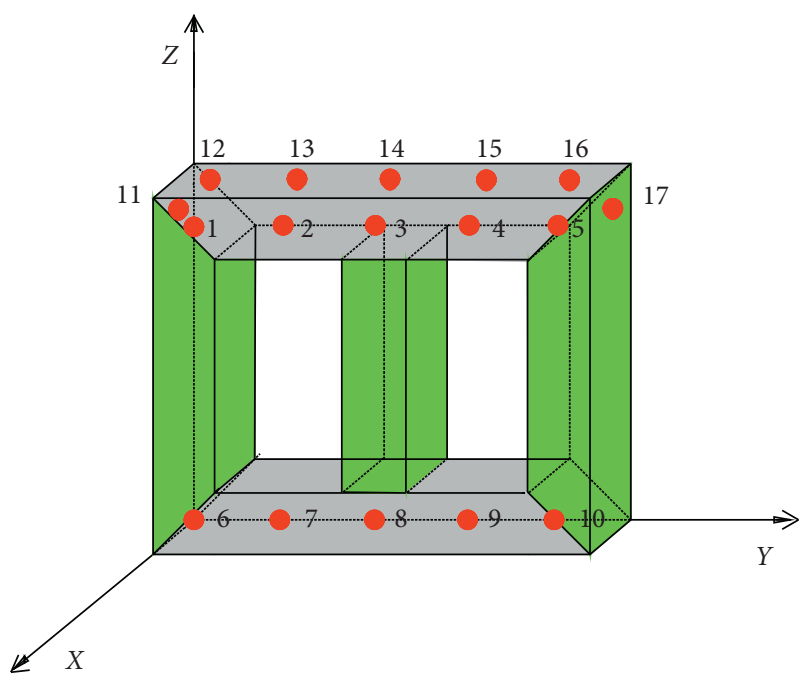

(a)

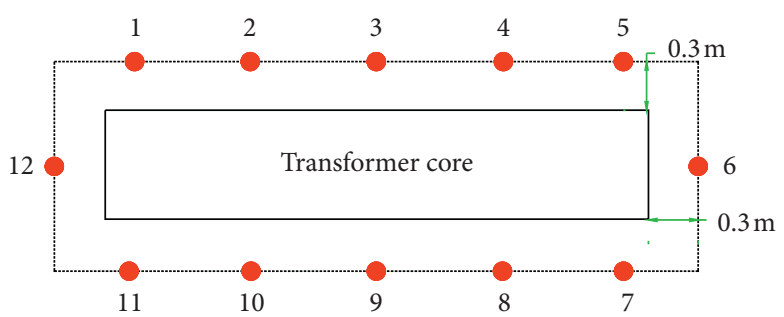

(b)

Figure 3: Noise and vibration measurement points. (a) Vibration measurement points (Nos. 1-10 in the vertical core plane and Nos. 11-17 in the parallel core plane). (b) Noise measurement points.
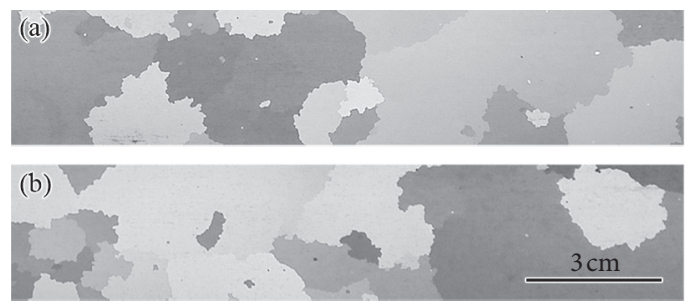

FIgURE 4: The microstructure of the central and edge seam regions in the core plane of SSS. (a) Central area. (b) Edge seam area.

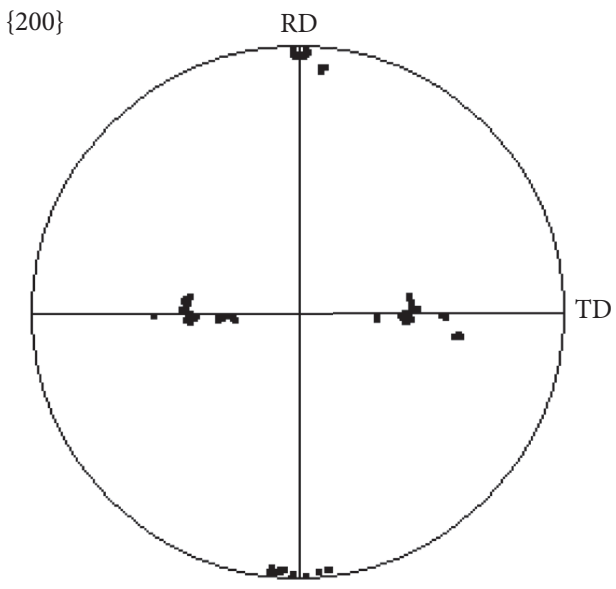

(a)

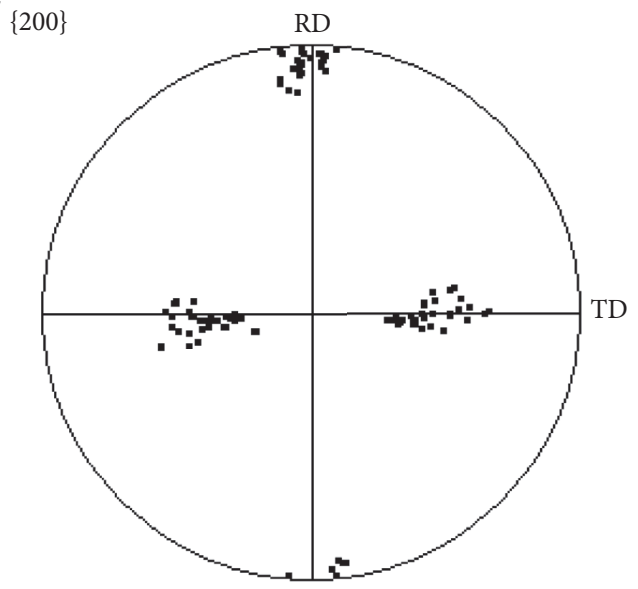

(b)

FIgURE 5: The texture of the center and edge seam areas in the core plane of SSS. (a) Central region. (b) Edge seam region.

magnetization saturation is reached, and the strain of magnetic domains also tends to be saturated. If the magnetic field intensity is further increased, the magnetization will increase slowly, and the variation behavior will no longer be consistent with the sample before saturation.
Figure 8 shows a time-domain variation curve of the SSS magnetostriction under different magnetic densities in one period. At the magnetic density of $1.7 \mathrm{~T}$, the curve is no longer smooth and becomes distorted. The Fourier spectrum analysis results are depicted in Figure 9. When the magnetic 


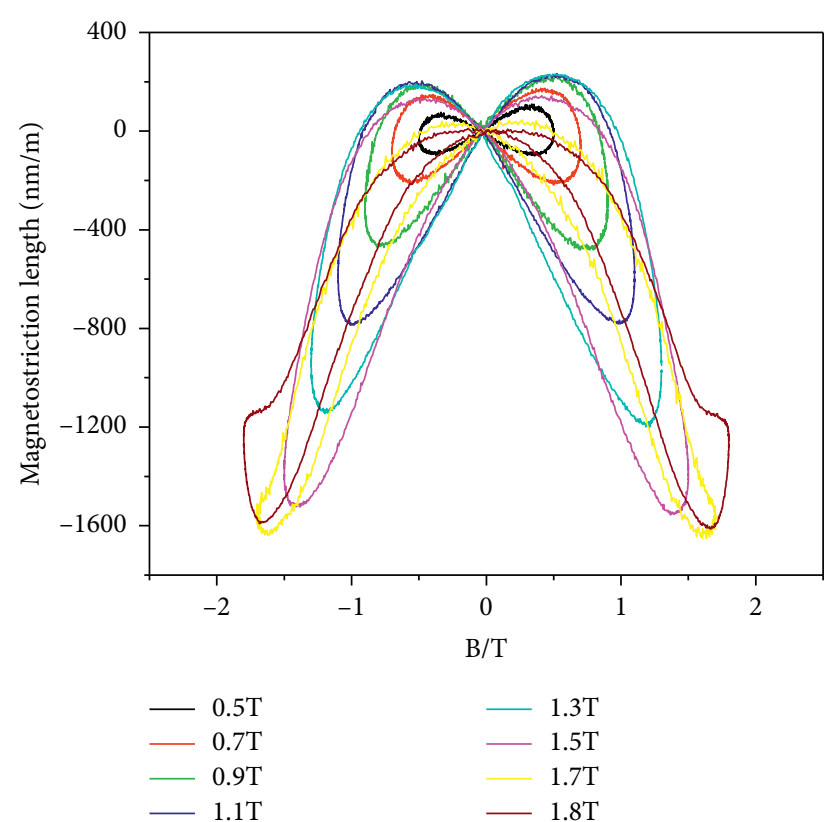

FIGURE 6: Magnetostrictive butterfly curves under different magnetic flux densities.

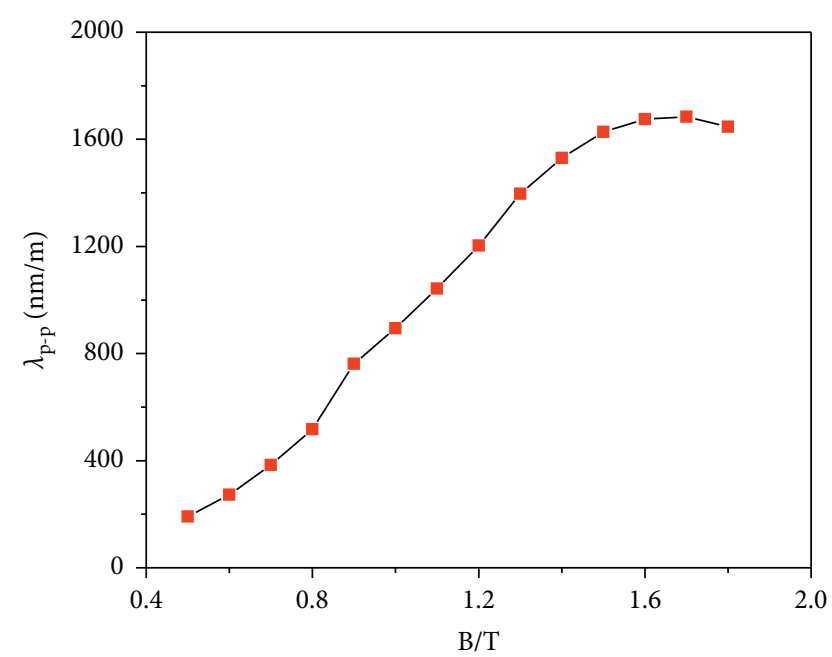

FIgURE 7: The magnetostrictive single-value curve.

flux density is less than $1.7 \mathrm{~T}$, the maximum magnetostrictive lengths are mainly concentrated at 0 and $100 \mathrm{~Hz}$. After $1.7 \mathrm{~T}$, the magnetostrictive lengths at frequencies of 200 and $300 \mathrm{~Hz}$ increased significantly.

Figure 10 depicts the butterfly curve of the grainoriented SSS in different directions at a magnetic density of 1.3 T under a $50 \mathrm{~Hz}$ sinusoidal periodic excitation magnetic field. It can be seen that the butterfly curves in different directions have a left-right symmetry, and magnetostriction directions are not the same, showing high magnetostrictive anisotropy characteristics. Along the rolling direction, the magnetostrictive value is the smallest. In the $45^{\circ}$ direction, the magnetostrictive value increases and shows shrinkage. In the vertical direction, the magnetostrictive value is the

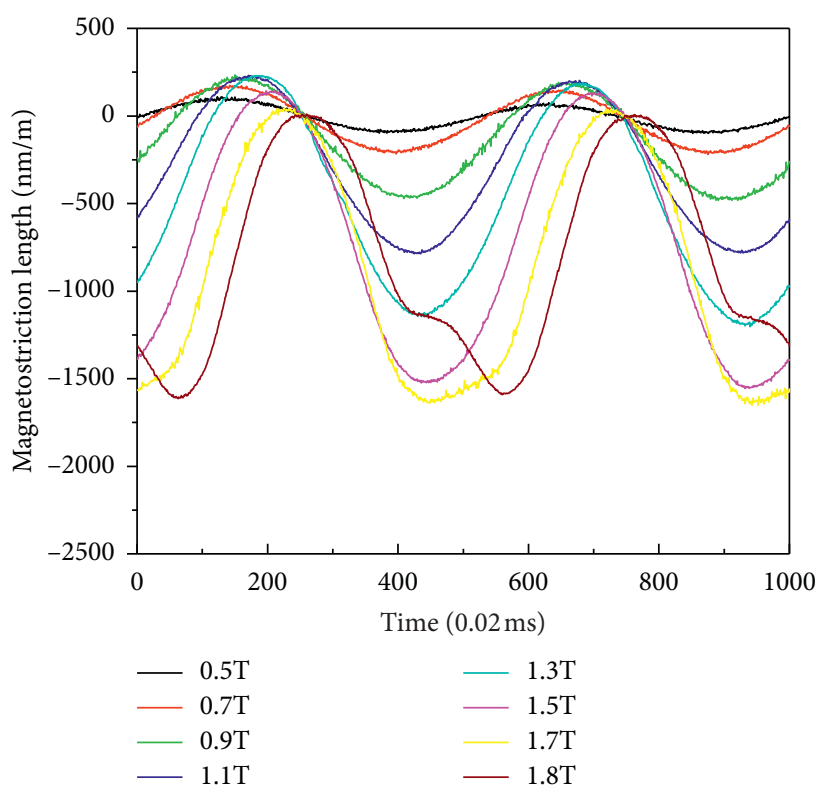

FIgURE 8: Time-domain waveforms of magnetostriction at different magnetic flux densities.
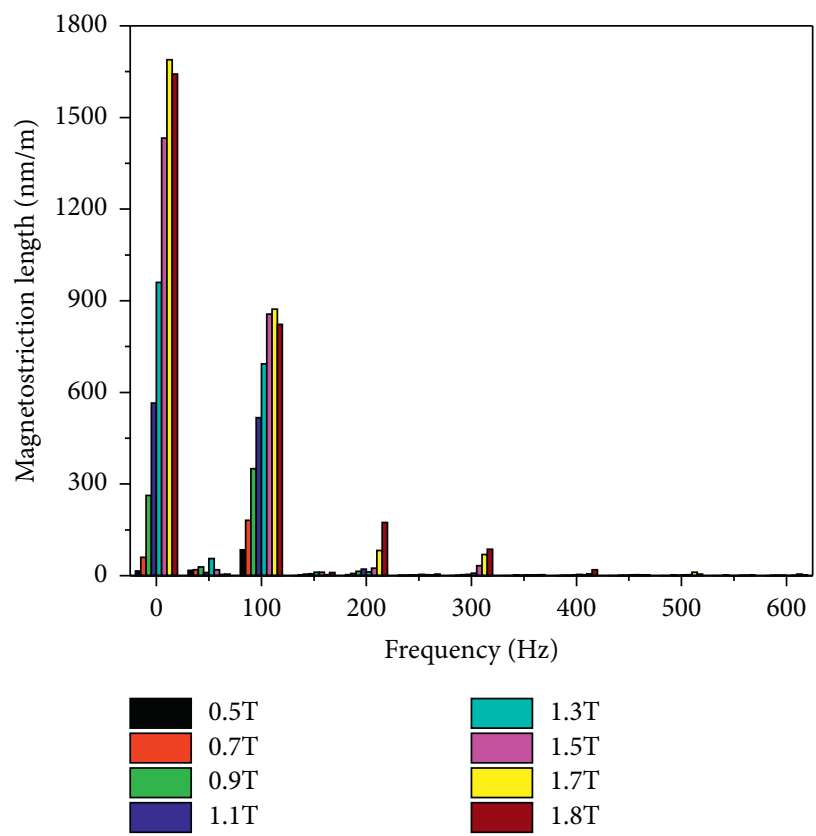

Figure 9: Magnetostrictive spectra at different magnetic flux densities.

largest and shows elongation. The maximum value of magnetostriction in the vertical direction exceeds that in the rolling direction by 35 times. The butterfly curves vertical to the rolling direction under different magnetic densities are omitted for brevity. Their variation pattern and magnetostrictive characteristics are the same as those along the rolling direction: they both increase in magnetostriction as the magnetic flux density increases until saturation. However, at the same magnetic flux density, the magnetostriction 


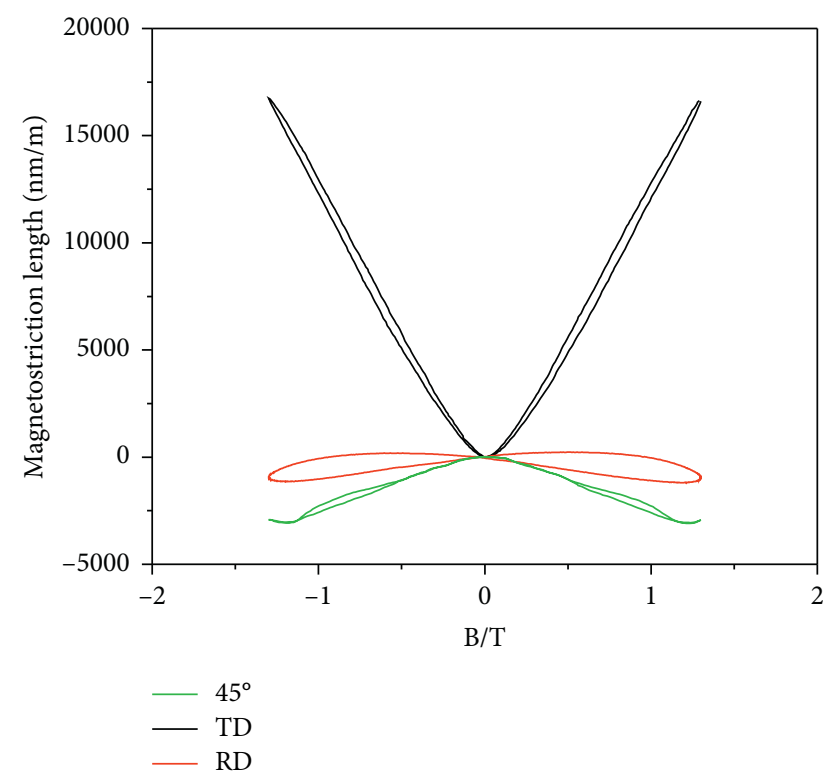

FIGURE 10: Butterfly curves of grain-oriented SSS under sinusoidal excitation of $1.3 \mathrm{~T}$ magnetic density in different directions.

of the grain-oriented SSS in the vertical direction is significantly larger than that in the parallel (rolling) direction.

Figure 11 shows the AWV noise curves of grainoriented SSS in different directions with different magnetic flux densities under $50 \mathrm{~Hz}$ sinusoidal periodic magnetic field excitation. Limited by the equipment power, the maximum field strength in the vertical and $45^{\circ}$ directions is $1.6 \mathrm{~T}$. It can be seen that the AWV noise level of grain-oriented SSS varies in different directions. Under the same magnetic density, the AWV noise is the smallest along the rolling direction, slightly increases in the $45^{\circ}$ direction, and is the largest in the vertical direction. The latter AWV noise is nearly twice higher than that in the rolling direction. The rate of increase of the AWV noise value of grain-oriented SSS is not affected by sample saturation. Before and after the sample is saturated, the rise of the AWV noise value is consistent with an increase in the magnetic flux density. Although the increase rate of the magnetostrictive peak-to-peak value $\lambda_{p-p}$ becomes smaller after saturation, it does not increase or even decrease slightly after the sample becomes saturated. However, with the emergence of the saturation phenomenon, the harmonic content contained in the magnetostrictive waveform increases, and the contribution to the AWV noise value is relatively large, so the AWV noise value increases.

\subsection{Vibration Characteristics of the Four-Corner Iron Core} Model. Figure 12 shows the vibration time domain and vibration spectrum of the four-corner iron core model SSS at a magnetic density of $1.3 \mathrm{~T}$. It can be seen that the time-domain vibration signal is not a standard sinusoidal waveform, but a distorted waveform superimposed by various frequency signals. However, it is still possible to determine the core vibration signal as a periodic signal with a frequency of $100 \mathrm{~Hz}$.

The main occurrence locations of vibration frequency peaks at each measurement point and the total effective value

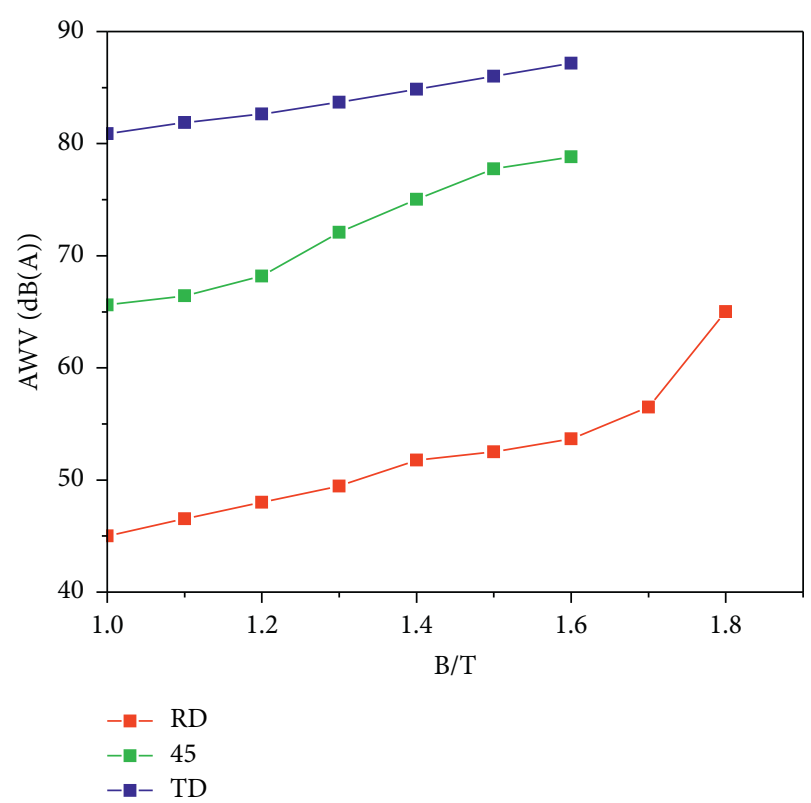

FIGURE 11: AWV noise curve of grain-oriented SSS in various directions.

of the vibration acceleration are shown in Table 1 . The main vibration peak frequencies in different directions are obviously different. In addition to the fundamental frequency, there are also well-pronounced high-frequency components, which are consistent with the theoretical analysis results. In addition, 450 and $650 \mathrm{~Hz}$ odd-order harmonics also appeared in the seam area (points 3 and 4), which may be caused by the combined effect of the cyclic excitation in the common area and the electromagnetic force between gaps. The effective values of vibration acceleration vertical to the core plane $\left(a_{1}\right.$ and $\left.a_{3}\right)$ are higher than those parallel to the core plane $\left(a_{2}, a_{4}\right.$, and $\left.a_{5}\right)$.

Figure 13 shows the vibration characteristics of measurement points 2, 3, and 5 under different magnetic densities. It can be seen that, with an increase in the magnetic density, the peak intensity of the main vibration frequency continues to increase, new frequency vibration peaks appear, and the vibration waveform becomes more complicated. The fundamental frequency vibration acceleration amplitude exhibits a linear relationship with the squared magnetic density, as shown in Figure 14. That is, the fundamental frequency vibration acceleration has a linear relationship with the squared voltage, which is consistent with the foregoing observation that the core vibration is proportional to the squared operating voltage.

Figure 15 shows the acceleration value of each measurement point under different magnetic densities. As the magnetic density increases, the acceleration value of each measuring point increases accordingly. It shows that the vibration vertical to the core plane is always higher than that parallel to it. In the same direction, the vibration in the central area of the SSS is significantly smaller than that in the seam area of the edge $\left(a_{1}<a_{3}\right.$ and $\left.a_{2}<a_{4}\right)$. In the plane parallel to the iron core, the vibration phase in the rolling direction $\left(a_{5}\right)$ is similar to that in the central area $\left(a_{2}\right)$ in the 

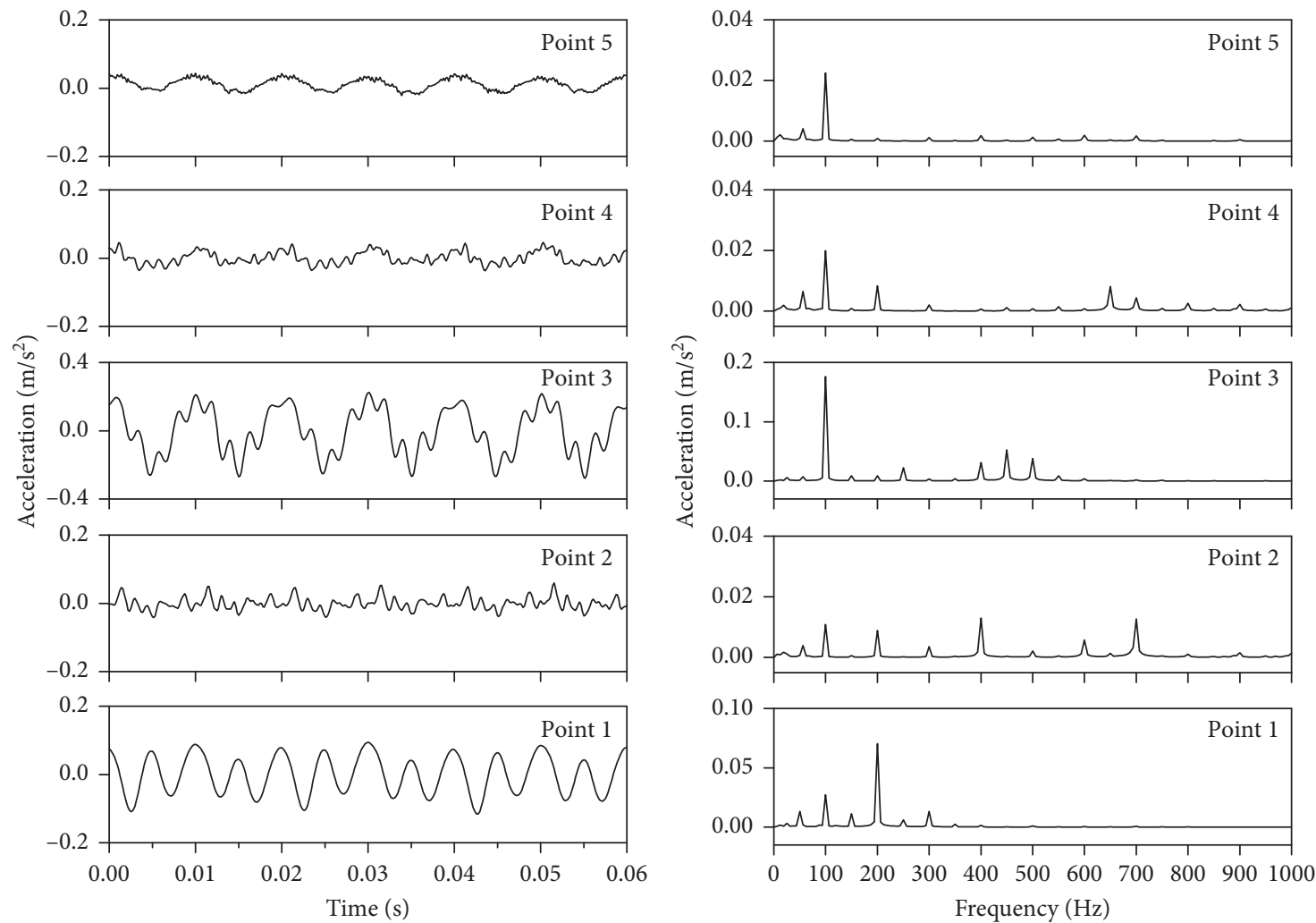

FIGURE 12: Vibration time-domain and spectrum plots of different measurement points at $1.3 \mathrm{~T}$ magnetic density. (a) Vibration timedomain diagram. (b) Vibration frequency-domain diagram.

TABLE 1: Vibration results of different measurement points under the magnetic density of $1.3 \mathrm{~T}$.

\begin{tabular}{lcc}
\hline Measurement point & Main vibration peak frequency $(\mathrm{Hz})$ & The effective value of vibration acceleration $\left(\mathrm{m} / \mathrm{s}^{2}\right)$ \\
\hline 1 & 100 and 200 & 0.072 \\
2 & $100,200,400$, and 700 & 0.042 \\
3 & 100 & 0.166 \\
4 & 100 and 200 & 0.041 \\
5 & 100 & 0.038
\end{tabular}

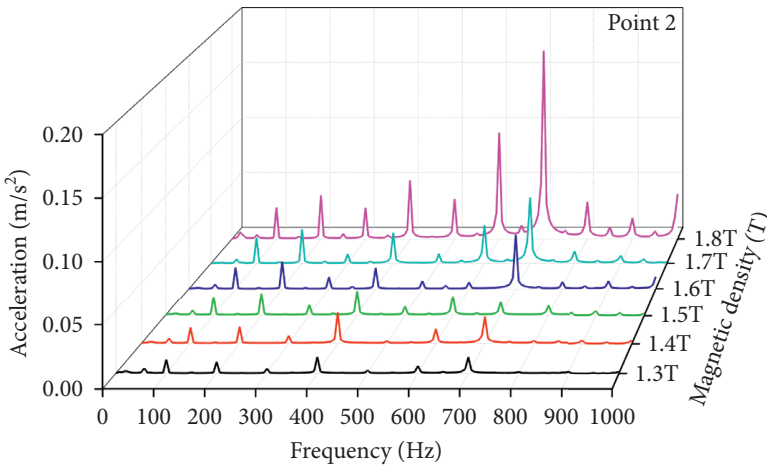

(a)

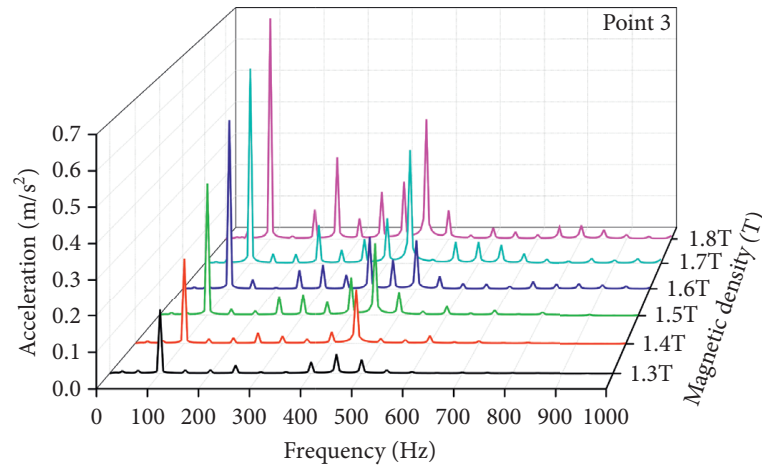

(b)

FIgURE 13: Continued. 


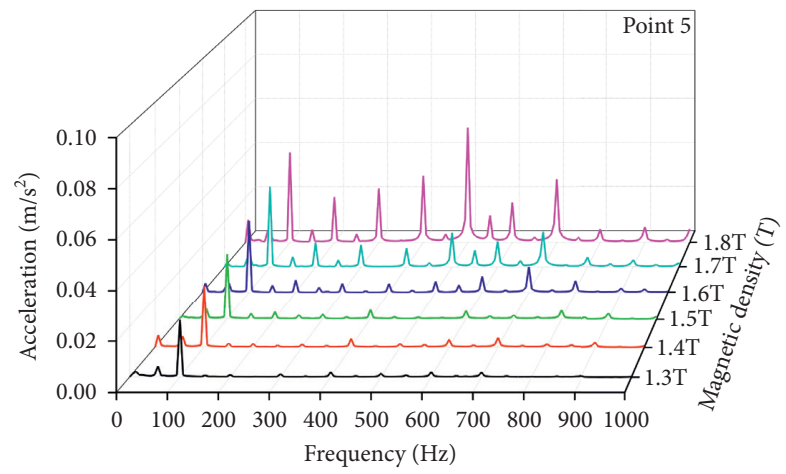

(c)

FIGURE 13: Vibration characteristics of measurement points 2, 3, and 5 under different magnetic densities.

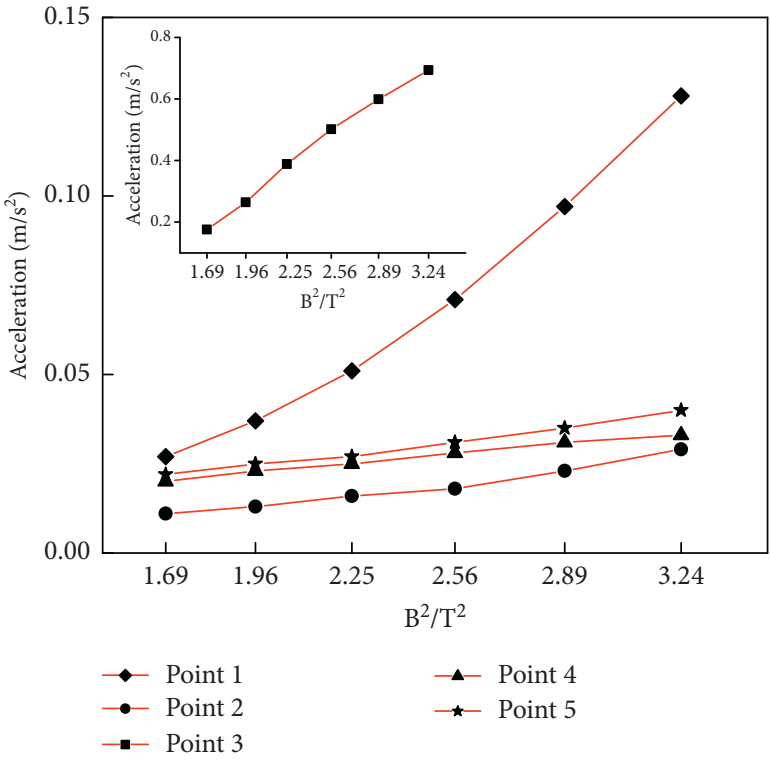

FIGURE 14: Relationship between the amplitude of fundamental frequency vibration acceleration and square of magnetic density.

vertical (rolling) direction at a small-scale magnetic density. But as the magnetic density increases, the vibration vertical to the rolling direction is gradually greater than that grainoriented along this direction.

As mentioned earlier, the microstructure and texture of various regions of SSS are not significantly different, in contrast to the SSS magnetostriction in different directions. Therefore, the main reason for the obvious difference between various regions is the SSS magnetostriction.

In the SSS central area, the magnetic field distribution is continuous and uniform, and the magnetic field direction is definite and consistent. In contrast, the magnetic field distribution in the edge seam area is intermittent, and the presence of rotating magnetic flux causes the scattering of the magnetic field direction in this area in multiple directions. At the same time, during the deformation process in the microstructure of the seam area, grains are deformed and rotate more freely because they are less hindered and

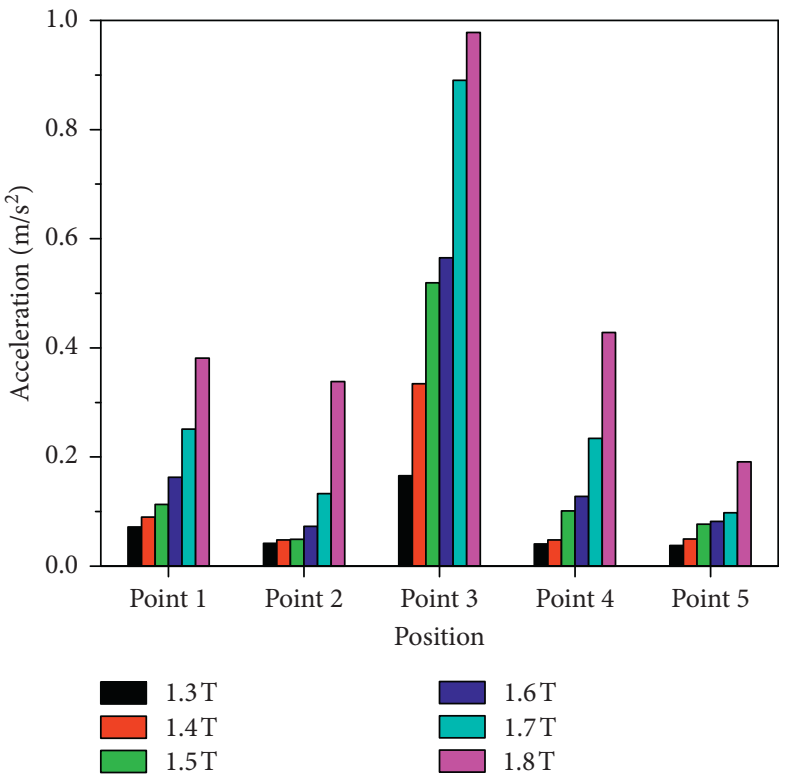

FIgURE 15: Acceleration values at various measurement points under different magnetic densities.

restrained by the surrounding grains. In addition, due to the mutual compression between the SSS in the common area, deformation behavior other than magnetostriction may occur. These factors cause the seam area to have completely different magnetostrictive characteristics from the central area, which will inevitably lead to low and high vibration parameters of the core central and seam areas, respectively, as well as differences in their vibration spectrum characteristics. At the same time, microdeformation vertical to the core plane is generated.

Under the magnetic field effect, the magnetostrictive direction of the core SSS mainly coincides with the SSS rolling direction and the magnetic field direction. However, both ends of the SSS are constrained by six degrees of freedom. Therefore, the magnetic field causes the SSS magnetostriction in its direction, but the material deformation cannot be released from both ends. It causes microbending and torsional deformation in the middle part 
of the SSS, and the component of this deformation in the direction vertical to the SSS plane will cause periodic deformation between laminations. This periodic microdeformation behavior induces vibrations in a direction vertical to the SSS plane. It can be seen that the core vibration in the direction vertical to the core plane is the result of the joint deformation of the edge seam area of the core SSS along this direction and the microdeformation between laminations in the central region. The vibration parallel to the core plane is the result of the SSS deformation in the magnetic field direction.

4.3. Modal, Noise, and Vibration Characteristics of the Actual Transformer Core. Figure 16 is a photo of a modal test of a $110 \mathrm{kV}$ transformer core. Modal test results are shown in Table 2. It can be seen that, in the vicinity of the natural frequency of the transformer core, it is easy to cause the equipment resonance and increase its overall vibration and noise levels.

Figure 17 shows the first six natural frequency mode characteristics of the transformer core. It can be seen that the first-order mode of the transformer core is mainly characterized by the vibration deformation of the overall structure and the local torsion as the auxiliary. The secondorder mode is mainly characterized by the bending and torsion deformation and twist deformation of the structure. The third-order mode is mainly characterized by the torsional deformation of the structure. The fourth-order mode is mainly characterized by the bending and torsion deformation of the structure. The vibration modes above the fifthorder one show the combined effect characteristics of torsional deformation and bending deformation. As the core structural order rises, its modal characteristics gradually change from bending deformation to multiorder bending and torsional deformation, showing more complex deformation characteristics.

The experimental field diagrams of the vibration and noise test of the $110 \mathrm{kv}$ transformer core components under no-load conditions and the frequency spectrum characteristics of some measurement points are shown in Figures 18 and 19 . The frequency of the vibration acceleration peak vertical to the core plane mainly appears at $100,200,300$, and $500 \mathrm{~Hz}$. There are also certain peaks at 50, 150, 250, 350, 400, $600,700,800$, and $900 \mathrm{~Hz}$. The frequency of the vibration acceleration peak parallel to the core plane mainly appears near 300 and $500 \mathrm{~Hz}$. There are also certain peaks at 100, 200, $400,600,700,800$, and $900 \mathrm{~Hz}$. The vibration spectrum characteristics of different positions and directions vary. The total effective value of the vibration acceleration at each point is shown in Figure 20. The amplitude of vibration in the direction parallel to the core plane is generally lower than that in the vertical direction. In the same direction, there is a gradually decreasing trend from both ends to the center. Due to the differences in the manufacturing process, capacity, and testing of the four-corner core model and the actual core products, the main vibration spectrum in each area is slightly different, but they are mainly based on the $100 \mathrm{~Hz}$ fundamental frequency and higher harmonic frequencies.

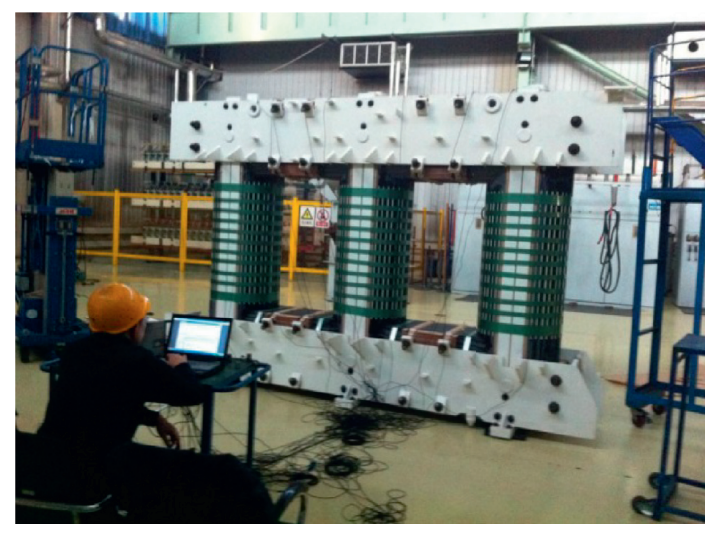

Figure 16: Modal test site of the transformer core.

TABLe 2: Modal test results of the transformer core.

\begin{tabular}{lc}
\hline Order & Experimental value $(\mathrm{Hz})$ \\
\hline 1 & 8.41 \\
2 & 23.61 \\
3 & 65.71 \\
4 & 100.86 \\
5 & 175.43 \\
6 & 203.6 \\
7 & 299.31 \\
8 & 344.62 \\
9 & 400.65 \\
10 & 493.95 \\
11 & 570.34 \\
12 & 592.06 \\
13 & 705.97 \\
14 & 755.49 \\
\hline
\end{tabular}

However, the vibration behavior is completely in accordance with the vibration behavior in different regions of the above four-corner core model.

The noise spectrum characteristics (vertical and parallel to the core plane direction) are depicted in Figures 21(a) and 21(b), respectively. The peak frequencies with the highest noise vertical to the core plane mainly appear near 100, 200, and $500 \mathrm{~Hz}$. There are also obvious noise peaks at such frequencies, such as $50,80,300,400$, and $600 \mathrm{~Hz}$. For the noise spectra parallel to the iron core plane, the highest noise peak frequencies mainly appear near 300 and $500 \mathrm{~Hz}$. There are also obvious noise peaks near such frequencies as 50,80 , 100, 200, 400, and $600 \mathrm{~Hz}$.

It can be seen that the core vibration and noise spectra in the same direction have significant acoustic vibration correlation characteristics. In the direction vertical to the core plane, the frequencies of the extreme amplitudes of vibration and noise mainly appear near 100, 200, and $500 \mathrm{~Hz}$. There are also certain peaks near such frequencies as 50,300, and $400 \mathrm{~Hz}$. In the direction parallel to the core plane, the frequencies of extreme amplitudes of vibration and noise mainly appear near 300 and $500 \mathrm{~Hz}$. Peaks also appeared near such frequencies as 100,200, and $400 \mathrm{~Hz}$. The noise at the relevant frequency is mainly attributed to the core vibration. 


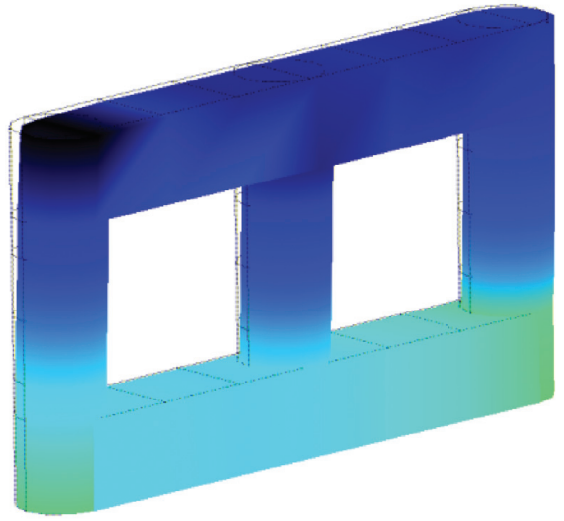

(a)

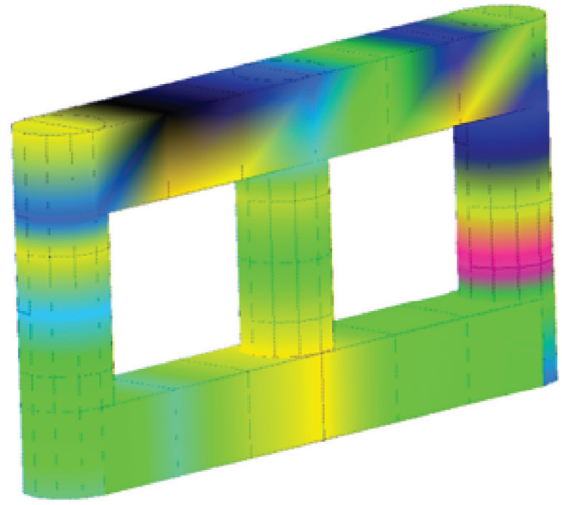

(d)

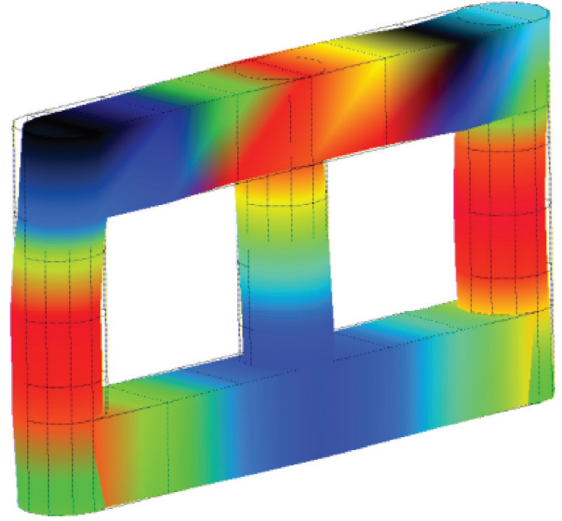

(b)

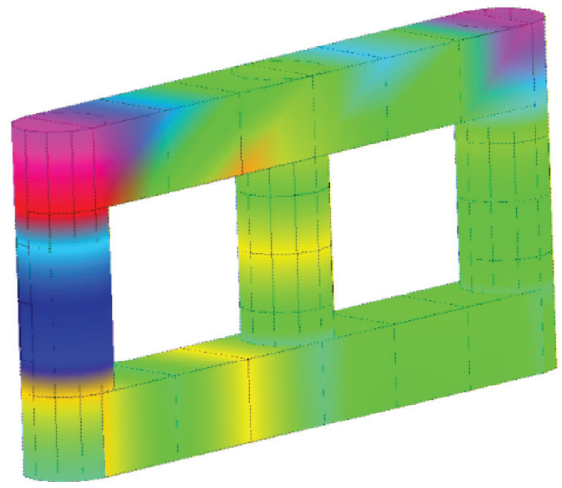

(e)

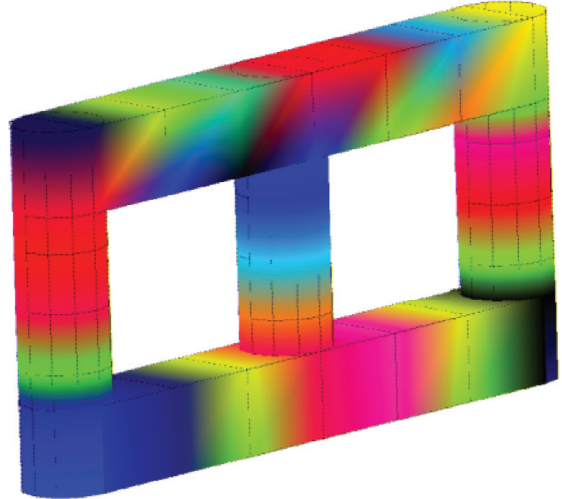

(c)

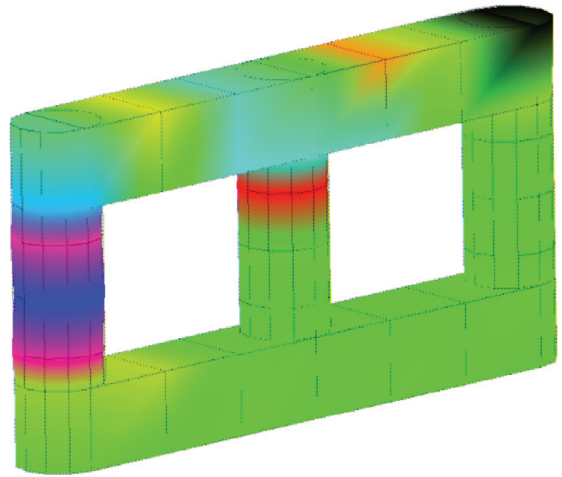

(f)

Figure 17: Test modal vibration modes of the transformer core. (a) First-order vibration mode. (b) Second-order vibration mode. (c) Thirdorder vibration mode. (d) Fourth-order vibration mode. (e) Fifth-order vibration mode. (f) Sixth-order vibration mode.

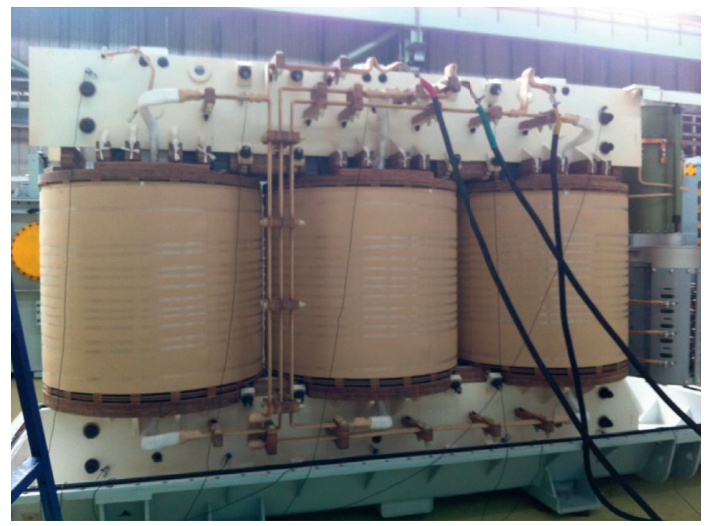

FIGURE 18: Noise and vibration tests of the core under no-load conditions.

Figure 22 shows the relationship between core modes, vibration, and noise. The first-order vibration of the core inherently includes frequencies near 50,100 , and $200 \mathrm{~Hz}$, and its mode characteristics are mainly bending and torsional deformation in the direction vertical to the core plane. The analysis of the vibration spectrum in two directions shows that only the former vibration direction has a maximum peak near frequencies of 100 and $200 \mathrm{~Hz}$. The noise spectrum also has peaks of maximum noise near 100 and $200 \mathrm{~Hz}$, indicating that the core modal characteristics strongly influence its vibration and noise characteristics, which may cause structural resonance at these frequencies. It can be seen that the core modal characteristics, vibration, and noise also have a close correlation.

Based on the above analysis, three low-noise core design points are given. One is to select a low magnetostrictive silicon steel sheet that matches the core compression force to 

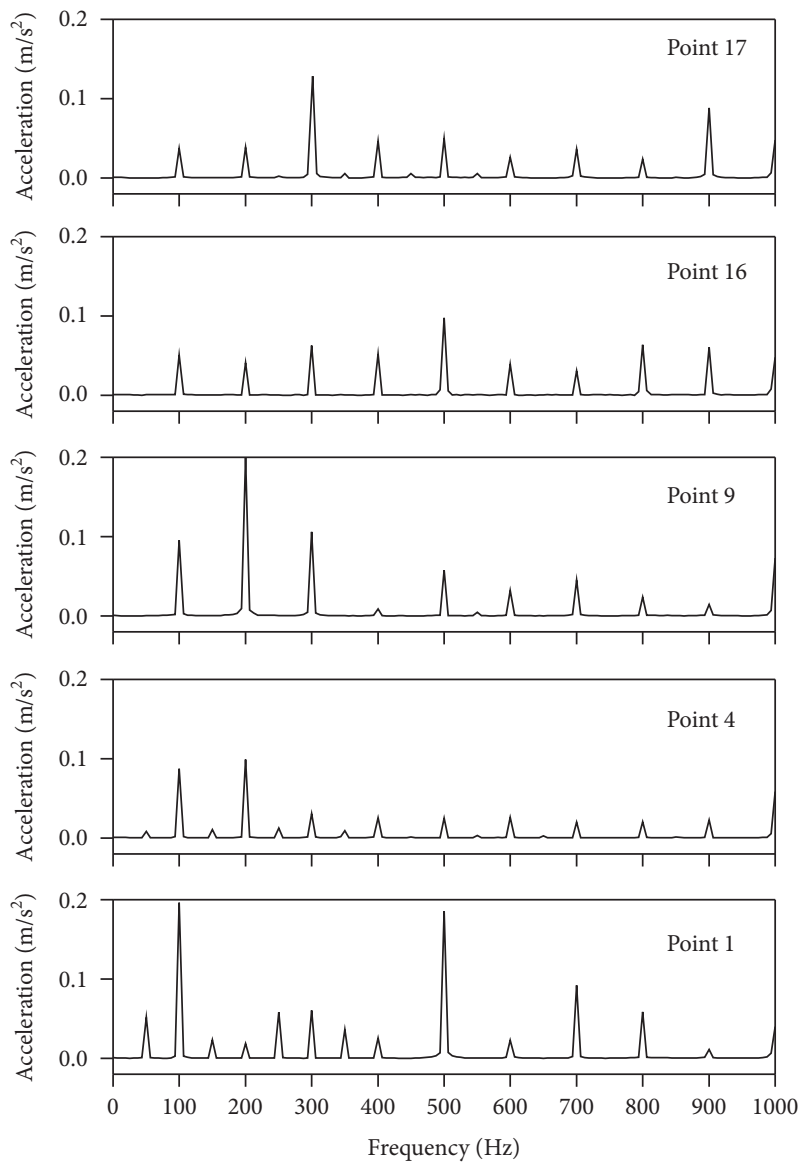

FIgURE 19: Peak vibration spectrum characteristics of the iron core.

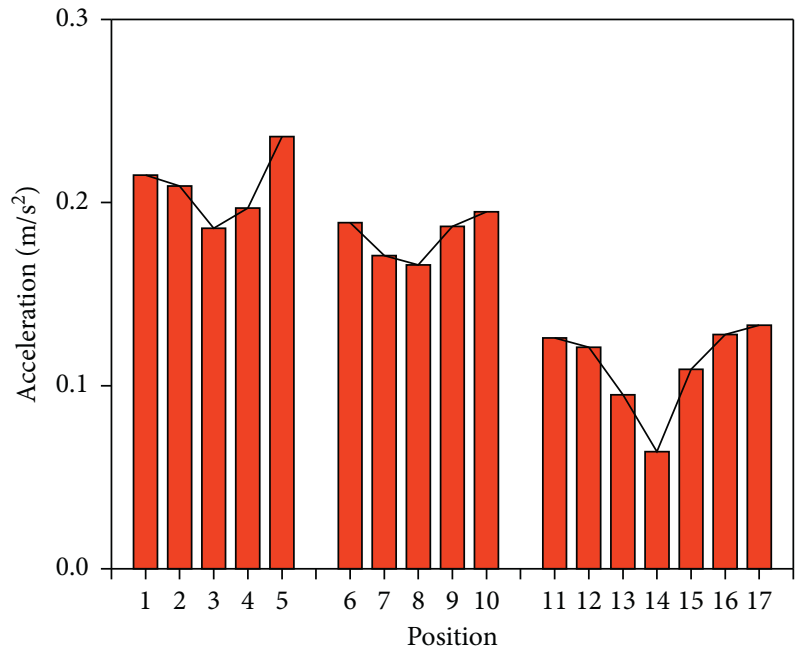

FIGURE 20: The total effective value of vibration acceleration at different measurement points of the iron core.

reduce the vibration amplitude in all directions of the core. The second is to change the lapping mode or spacing of the core seam area to reduce the vibration amplitude at the seam areas. The third is to use noise-reducing damping materials or improve the structure to change the modal characteristics of the core to keep it away from the main operating frequency band in the energized state. Avoid the occurrence of equipment resonance under the power-on operation state, and reduce the damage ability of the resonance to the equipment, thereby ensuring the safe operation of the equipment and improving the environmental protection level of the equipment. 


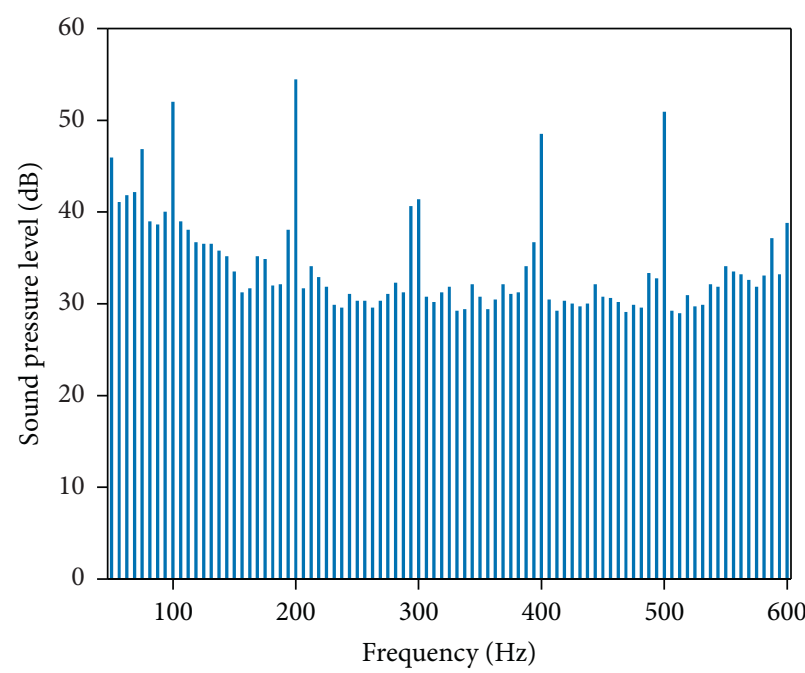

(a)

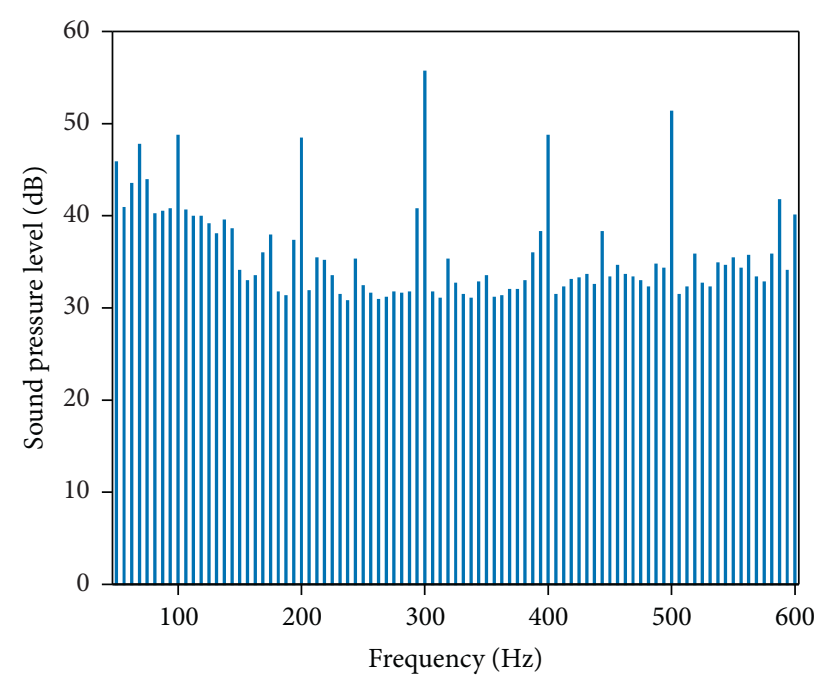

(b)

Figure 21: Spectrum characteristics of core noise. (a) Vertical to the core plane. (b) Parallel to the core plane.

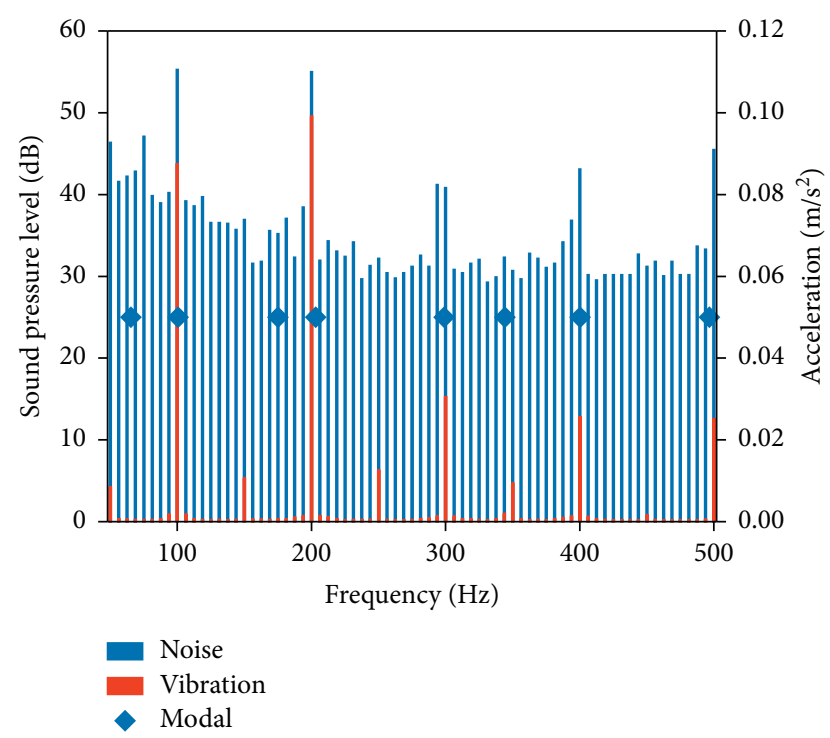

FIGURE 22: Correlation analysis of vibration, noise, and modal points of core components.

\section{Conclusions}

In this paper, the magnetostrictive characteristics of grainoriented silicon steel sheets (SSS) were tested. A four-corner core model platform was constructed to simulate the firstlevel vibration of the core. Finally, noise, vibration, and modal tests were performed on the actual $110 \mathrm{kV}$ core product. This work provided a detailed analysis of the core vibration mechanism from the material to the product. The main conclusions are as follows:

(1) The texture structure characteristics and crystal orientation distribution of the oriented SSS's central and edge seam areas are consistent. As the magnetic flux density of grain-oriented SSS increases, the magnetostriction in each direction grows until saturation, and the AWV noise value continues to increase without being affected by saturation. At the same magnetic flux density, the magnetostriction vertical to the rolling direction is significantly larger than that in the rolling direction, showing significant anisotropy.

(2) There are significant differences in the vibration of various areas of the four-corner core model. Vibrations vertical to the core plane exceed those parallel to it. Vibration in the central area in the same plane is significantly lower than that in the edge seam area. This difference is caused by the different microscopic deformation contributed by various regions with completely different magnetostrictive characteristics.

(3) The vibration behavior of the actual $110 \mathrm{kV}$ iron core product is shown to be the same as that of the fourcorner iron core model. In the same direction, there is a gradually decreasing trend from both seams to the center, and the vibration and noise spectra have significant acoustic vibration correlation characteristics. Its modal values also have a close correlation with its vibration and noise characteristics.

In the future, the vibration amplitude of the core can be reduced by choosing the low magnetostrictive silicon steel sheet which matches the core compression force, changing the lapping mode or spacing of the core seam area, increasing the noise-reducing damping materials, or improving the structure.

\section{Data Availability}

The data used to support the findings of this study are available from the corresponding author upon request.

\section{Conflicts of Interest}

The authors declare that there are no conflicts of interest regarding the publication of this paper. 


\section{Acknowledgments}

This work was supported by the Scientific and Technological Project of State Grid Corporation of China (Grant no. 5500-202058312A-0-0-00).

\section{References}

[1] IEEE Standard, "IEEE Guide for sound level abatement and determination for liquid-immersed power transformers and shunt reactors rated over $500 \mathrm{kVA}$," IEEE Standard, vol. 57, pp. 136-2000, 2005.

[2] International Electrotechnical Commission, "Power transformers: Part 10: determination of sound levels," International Electrotechnical Commission, vol. 10, 2005.

[3] CIGRE Working Group 14, "26 Report. High voltage direct current (HVDC) substation audible noise," CIGRE Brochure, vol. 202, 2004.

[4] L. Cheng, F. Y. Yang, G. Ma et al., "Magnetic performance of grain-oriented silicon steel under complex working conditions and material selection for transformer core," Materials Reports (B), vol. 33, no. 7, pp. 2413-2418, 2019.

[5] D. Z. Chen, W. L. Zhao, B. D. Bai et al., "Analysis and experiment of transformer vibration and noise considering electrical steel sheet magnetostriction," International Journal of Applied Electromagnetics and Mechanics, vol. 52, no. 3-4, pp. 1477-1484, 2016.

[6] Z. Wang, Y. L. Zhang, D. H. Zhang et al., "Modeling of magnetostrictive property of electrical steel sheet under vectorial excitation," IEEE Transactions on Magnetics, vol. 55, no. 6, Article ID 7300304, 2019.

[7] A. J. Moses, P. I. Anderson, and T. Phophongviwat, "Localized surface vibration and acoustic noise emitted from laboratoryscale transformer cores assembled from grain-oriented electrical steel," IEEE Transactions on Magnetics, vol. 52, no. 10, pp. 1-15, 2016.

[8] S. Okabe, Y. Ishigaki, and T. Omura, "Vibration mode and vibration shape under excitation of a three-phase model transformer core," AIP Advances, vol. 8, Article ID 047902, 2018.

[9] Y.-H. Chang, C.-H. Hsu, H.-L. Chu, and C.-P. Tseng, "Magnetomechanical vibrations of three-phase three-leg transformer with different amorphous-cored structures," IEEE Transactions on Magnetics, vol. 47, no. 10, pp. 27802783, 2011.

[10] C. H. Hsu, Y. M. Huang, M. F. Hsieh et al., "Transformer sound level caused by core magnetostriction and winding stress displacement variation," AIP Advances, vol. 7, Article ID 056681, 2017.

[11] A. Moses, "Measurement of magnetostriction and vibration with regard to transformer noise," IEEE Transactions on Magnetics, vol. 10, no. 2, pp. 154-156, 1974.

[12] Z. D. Zhang, S. R. Xu, J. G. Zhang et al., "Study on vibration noise signal characteristics of $10 \mathrm{kv}$ distribution transformer under different load conditions," IOP Conference Series: Materials Science and Engineering, vol. 486, Article ID 012033, 2019.

[13] C. Liu, X. Li, and X. Li, "Simulating the vibration increase of the transformer iron core due to the DC bias," International Journal of Applied Electromagnetics and Mechanics, vol. 55, no. 3, pp. 423-433, 2017.

[14] K. Bouayed, L. Mebarek, V. Lanfranchi, J.-D. Chazot, R. Marechal, and M.-A. Hamdi, "Noise and vibration of a power transformer under an electrical excitation," Applied Acoustics, vol. 128, pp. 64-70, 2017.

[15] Y. C. Ma, J. Mo, G. Z. Wang et al., "Test and Experimental Study on vibration and noise characteristics of distribution transformer," IOP Conference Series: Earth and Environmental Science, vol. 526, Article ID 012136, 2020.

[16] P. N. Zhang, L. Lin, Z. G. Cheng et al., "Study on vibration of iron core of transformer and reactor based on maxwell stress and anisotropic magnetostriction," IEEE Transactions on Magnetics, vol. 55, no. 2, Article ID 9400205, 2019.

[17] F. Zhang, S. Ji, Y. Shi, C. Zhan, and L. Zhu, "Investigation on vibration source and transmission characteristics in power transformers," Applied Acoustics, vol. 151, pp. 99-112, 2019.

[18] X. Liu, Y. Yang, Y. Huang, and A. Jadoon, "Vibration characteristic investigation on distribution transformer influenced by DC magnetic bias based on motion transmission model," International Journal of Electrical Power \& Energy Systems, vol. 98, pp. 389-398, 2018.

[19] D. Chi-Phi and P. Anh-Tuan, "Study on vibrations and noises in the amorphous steel core transformer caused by magnetostriction effect," 9th International Conference on Robotic, Vision, Signal Processing and Power Applications, vol. 398, pp. 827-833, 2017.

[20] M. Javorski, G. Cepon, J. Slavic, and M. Boltezar, "A generalized magnetostrictive-forces approach to the computation of the magnetostriction-induced vibration of laminated steel structures," IEEE Transactions on Magnetics, vol. 49, no. 11, pp. 5446-5453, 2013.

[21] L. K. Xie, C. M. Chen, Y. Gao et al., "Study on noise attenuation laws and control methods of the transformer," $A d$ vanced Materials Research, vol. 610-613, pp. 2530-2534, 2013.

[22] S. G. Ghalamestani, L. Vandevelde, J. J. J. Dirckx et al., "Magnetostrictive vibrations model of a three-phase transformer core and the contribution of the fifth harmonic in the grid voltage," Journal of Applied Physics, vol. 115, Article ID 17A316, 2014.

[23] J. Z. Hu, D. C. Liu, Q. F. Liao et al., "Electromagnetic vibration noise analysis of transformer windings and core," IET Electric Power Applications, vol. 10, no. 4, pp. 251-257, 2016.

[24] B. Zhang, N. Yan, J. M. Du et al., "A novel approach to investigate the core vibration in power transformers," IEEE Transactions on Magnetics, vol. 54, no. 11, p. 8400804, 2018.

[25] M. Y. Liu, O. Hubert, X. Mininger et al., "Reduction of power transformer core noise generation due to magnetostrictioninduced deformations using fully coupled finite-element modeling optimization procedures," IEEE Transactions on Magnetics, vol. 53, no. 8, Article ID 8400511, 2017.

[26] Q. Li, X. Wang, L. Zhang, J. Lou, and L. Zou, "Modelling methodology for transformer core vibrations based on the magnetostrictive properties," IET Electric Power Applications, vol. 6, no. 9, pp. 604-610, 2012.

[27] S. Wu, W. Huang, F. Kong et al., "Extracting power transformer vibration features by a time-scale-frequency analysis method," Journal of Electromagnetic Analysis and Applications, vol. 2, no. 1, pp. 31-38, 2010.

[28] S. C. Ji, Y. F. Luo, and Y. M. Li, "Research on extraction technique of transformer core fundamental frequency vibration based on OLTC," IEEE Transactions on Power Delivery, vol. 2l, no. 4, pp. 1981-1988, 2006.

[29] C. Bartoletti, M. Desiderio, D. DiCarlo et al., "Vibro-acoustic techniques to diagnose power transformers," IEEE Transactions on Power Delivery, vol. 19, no. 1, pp. 221-229, 2004.

[30] C. K. Mechefske, "Correlating power transformer tank vibration characteristics to winding looseness," Insight: Non- 
destructive Testing and Condition Monitoring, vol. 37, no. 8, pp. 599-604, 1995.

[31] V. Dlamini, R. Naidoo, and M. Manyage, "A non-intrusive method for estimating motor efficiency using vibration signature analysis," International Journal of Electrical Power \& Energy Systems, vol. 45, pp. 184-190, 2013.

[32] T. Nakase, M. Nakano, K. Fujiwara, and N. Takahashi, "Measuring system for magnetostriction of silicon steel sheet under AC excitation using optical methods," IEEE Transactions on Magnetics, vol. 34, no. 4, pp. 2072-2074, 1998. 